\title{
Carbohydrate nutrition and risk of adiposity-related cancers: results from the Framingham Offspring cohort (1991-2013)
}

\author{
Nour Makarem ${ }^{1}$, Elisa V. Bandera ${ }^{2,3}$, Yong Lin ${ }^{2,3}$, Paul F. Jacques ${ }^{4}$, Richard B. Hayes ${ }^{5}$ and \\ Niyati Parekh ${ }^{5,6 *}$ \\ ${ }^{1}$ Department of Medicine, Columbia University Medical Center, 51 Audubon Avenue, Suite 501, New York, NY 10032, USA \\ ${ }^{2}$ Rutgers School of Public Health, Rutgers The State University of New Jersey, 683 Hoes Lane West, Piscataway, NJ 08854, USA \\ ${ }^{3}$ Rutgers Cancer Institute of New Jersey, 195 Little Albany Street, New Brunswick, NJ 08903-2681, USA \\ ${ }^{4}$ Friedman School of Nutrition Science and Policy, Jean Mayer USDA Human Nutrition Research Center on Aging, Tufts \\ University, 711 Washington Street, Boston, MA 02111, USA \\ ${ }^{5}$ Department of Population Health, NYU Langone School of Medicine, 227 East 30th Street, 7th Floor, New York, NY 10016, USA \\ ${ }^{6}$ College of Global Public Health, New York University, 715-719 Broadway, Room 1220, New York, NY 10003, USA \\ (Submitted 24 November 2016 - Final revision received 29 April 2017 - Accepted 31 May 2017 - First published online 29 June 2017)
}

\begin{abstract}
Higher carbohydrate intake, glycaemic index (GI), and glycaemic load (GL) are hypothesised to increase cancer risk through metabolic dysregulation of the glucose-insulin axis and adiposity-related mechanisms, but epidemiological evidence is inconsistent. This prospective cohort study investigates carbohydrate quantity and quality in relation to risk of adiposity-related cancers, which represent the most commonly diagnosed preventable cancers in the USA. In exploratory analyses, associations with three site-specific cancers: breast, prostate and colorectal cancers were also examined. The study sample consisted of 3184 adults from the Framingham Offspring cohort. Dietary data were collected in 1991-1995 using a FFQ along with lifestyle and medical information. From 1991 to 2013, 565 incident adiposity-related cancers, including 124 breast, 157 prostate and sixty-eight colorectal cancers, were identified. Cox proportional hazards models were used to evaluate the role of carbohydrate nutrition in cancer risk. GI and GL were not associated with risk of adiposity-related cancers or any of the site-specific cancers. Total carbohydrate intake was not associated with risk of adiposity-related cancers combined or prostate and colorectal cancers. However, carbohydrate consumption in the highest $v$. lowest quintile was associated with $41 \%$ lower breast cancer risk (hazard ratio (HR) $0 \cdot 59$; $95 \%$ CI $0 \cdot 36,0.97)$. High-, medium- and low-GI foods were not associated with risk of adiposity-related cancers or prostate and colorectal cancers. In exploratory analyses, low-GI foods, were associated with $49 \%$ lower breast cancer risk (HR $0.51 ; 95 \%$ CI 0.32 , 0.83 ). In this cohort of Caucasian American adults, associations between carbohydrate nutrition and cancer varied by cancer site. Healthier low-GI carbohydrate foods may prevent adiposity-related cancers among women, but these findings require confirmation in a larger sample.
\end{abstract}

Key words: Carbohydrate intakes: Glycaemic index: Glycaemic load: Adiposity-related cancers: Framingham Offspring cohort

Cancer is a major cause of morbidity and mortality in the $\mathrm{USA}^{(1)}$. The high incidence rates of this disease continue to be stable for women and have declined only slightly for men over the past 5 years $^{(1)}$. A number of cancer types are now linked to overweight and obesity and are therefore termed 'adiposity-related ${ }^{,(2,3)}$. Adiposity-related cancers include the most commonly diagnosed cancers in the USA such as female, gastrointestinal, genitourinary, haematologic and other cancers $^{(2,3)}$ and account for more than 1.2 million of the projected 1.7 million incident cancers in $2017^{(1)}$. Due to the high economic costs associated with the management of these cancers $^{(4)}$, primary prevention by altering modifiable risk factors will probably be the most effective way of reducing the cancer burden at present.

Hyperglycaemia, hyperinsulinaemia and insulin resistance play a role in the aetiology of adiposity-related cancers ${ }^{(5)}$. Moreover, obesity, diabetes and the metabolic syndrome, all of which are characterised by glucose and insulin dysregulation, are established risk factors for cancer ${ }^{(6-8)}$. Dietary carbohydrates are the main dietary component impacting blood glucose and insulin levels and have also been linked to obesity risk and various measures of body adiposity ${ }^{(9,10)}$. However, their influence on chronic disease risk, particularly cancer, may vary by both quantity and type of carbohydrates consumed.

Abbreviations: FHS, Framingham Heart Study; FOS, Framingham Offspring; GI, glycaemic index; GL, glycaemic load; HR, hazard ratio; WC, waist circumference.

* Corresponding author: N. Parekh, fax +1 212998 4194, email niyati.parekh@nyu.edu 
Dietary glycaemic index (GI) is an index for ranking carbohydrate-containing foods based on their effect on blood glucose concentrations that serves as a measure of carbohydrate quality ${ }^{(11,12)}$. It provides a numerical evidence-based index of postprandial glycaemia by comparing available carbohydrates gram-for-gram in foods to a standard source, either glucose or white bread. Dietary glycaemic load (GL), on the other hand, is a ranking system for the carbohydrate content of food that takes into account the portion size in addition to the GI, and therefore serves as a measure of both average quantity and quality of carbohydrates ${ }^{(13)}$. The rise in blood glucose after a meal, influenced by dietary GI and GL, is linked to a rise in insulin ${ }^{(12)}$. Higher circulating concentrations of insulin result in the activation of the insulin-signalling pathway that dictates the activity of a network of proteins that increase the risk of cancer ${ }^{(5)}$. According to the World Cancer Research Fund \& American Institute for Cancer Research (WCRF/AICR) ${ }^{(14)}$, GI and GL have predicted risks of type 2 diabetes and $\mathrm{CHD}$ and related biomarkers, which are risk factors for cancer. These findings suggest that GI and GL may be useful markers in the context of cancer prevention.

Epidemiological evidence on whether total carbohydrate intake and dietary GI and GL play a role in cancer risk is contradictory ${ }^{(15-20)}$. Although a null association has been observed for some cancer sites ${ }^{(17,19,20)}$, other studies are indicative of a role of GI but not GL in cancer risk ${ }^{(15)}$ and vice versa ${ }^{(18)}$. The WCRF/AICR indicate that epidemiological evidence thus far is too inconsistent to draw any conclusions or make dietary recommendations based on the intertwined concepts of GI and GL ${ }^{(14)}$. To clarify these associations, we present results from a prospective cohort study, using the Framingham Offspring (FOS) cohort, that evaluated the associations between total carbohydrate intake, GI and GL in relation to risk of adiposity-related cancers combined and three of the most common site-specific cancers in the USA: breast, prostate and colorectal cancers. We restricted our analyses to adiposityrelated cancers, because these cancers are hypothesised to be lifestyle related and are hence most likely to benefit from dietary modification ${ }^{(14)}$. In exploratory analyses, this study has also assessed the impact of the main carbohydrate food sources in the US diet and of high-, moderate- and low-GI foods on the risk of these cancers.

\section{Methods}

\section{Study population and analysis data set}

Existing data from the Framingham Heart Study (FHS), a prospective study in Framingham, Massachusetts designed to study CVD epidemiology ${ }^{(21)}$, were used for these analyses. The analytic sample consisted of 3184 men and women from the FOS cohort, which includes the offspring of the original cohort of the FHS and their spouses. Between 1971 and 1975, 5124 participants were enrolled into the FOS, clinical and medical exams have been conducted, on average, every 4 years to collect medical, lifestyle and anthropometric data ${ }^{(22)}$. During the fifth examination cycle, which occurred between 1991 and 1995, the collection of dietary data were initiated and was available for 3418 participants.
Only dietary data from examination 5 were used, because a previous analysis on trends in carbohydrate consumption in the FOS did not reveal any clinically significant changes over time ${ }^{(23)}$. Participants were excluded if reported energy intakes were outside the ranges of $2510-16736$ and $2510-17573 \mathrm{~kJ} / \mathrm{d}$ for women and men, respectively ( $n$ 67), in consistency with the criteria for 'plausible intakes' as previously published in the FHS $^{(24)}$. In addition, participants who left $\geq 13$ food items blank on the FFQ were excluded ( $n$ 31). Participants with a history of adiposity-related cancer at or before examination $5(n 134)$ and pregnant women at examination 5 ( $n$ 2) were also excluded. Therefore, the final analytic data set included 3184 participants (Fig. 1). All procedures involving human subjects were approved by the Institutional Review Board for Research with Human Subjects at New York University (no. 10-7319). The FHS was conducted according to the Declaration of Helsinki guidelines, and written informed consent was obtained from all subjects enrolled in the FHS by FHS investigators.

\section{Assessment of dietary intake}

Usual dietary intake for the previous year was assessed at the fifth examination cycle (1991-1995) using the validated 126-item Harvard semi-quantitative $\mathrm{FFQ}^{(25)}$. This FFQ queried the frequency consumption of food items with standard serving sizes ranging from never or $<1$ serving/month to $>6$ servings $/ \mathrm{d}^{(25)}$. It also included separate questions to assess the use of vitamin and mineral supplements. FFQ were mailed to participants before the examination, and participants were asked to bring the FFQ with them for revision by trained personnel at the study visit to ensure accuracy. Nutrient intakes were calculated by multiplying the reported frequency of consumption of foods by the nutrient content of the specified portion using the US Department of Agriculture nutrient database ${ }^{(25)}$

The main dietary exposures of interest for these analyses were total carbohydrate intake (\% energy) in addition to dietary GL and GI, which were energy-adjusted by using the multivariable method. The validity of this FFQ has been examined in several population groups for nutrients and foods ${ }^{(25,26)}$. There appears to be a moderate correlation of 0.69 and 0.45 when comparing total carbohydrate intake from the FFQ to intake from multiple diet records in men and women, respectively ${ }^{(25,26)}$. In addition, dietary GI and GL, estimated from this FFQ have been associated with plasma TAG concentrations, a biomarker known to respond to carbohydrate intake, as an indirect measure of validity ${ }^{(27)}$.

\section{Ascertainment of exposure variables}

Glycaemic index. GI represents the incremental area under the 2-h blood glucose response curve (AUC) induced by $50 \mathrm{~g}$ of carbohydrate from a specific food and is calculated as the percentage of the area produced by the same amount of carbohydrates from a standard source, either glucose or white bread $^{(11)}$. In the FHS, the GI of individual foods from the FFQ was acquired from published estimates (approximately $53 \%)^{(13)}$ or imputed when necessary by matching similar foods based on energy content, carbohydrate, sucrose, fat and dietary fibre content (approximately 28\%). The remaining food items on the 


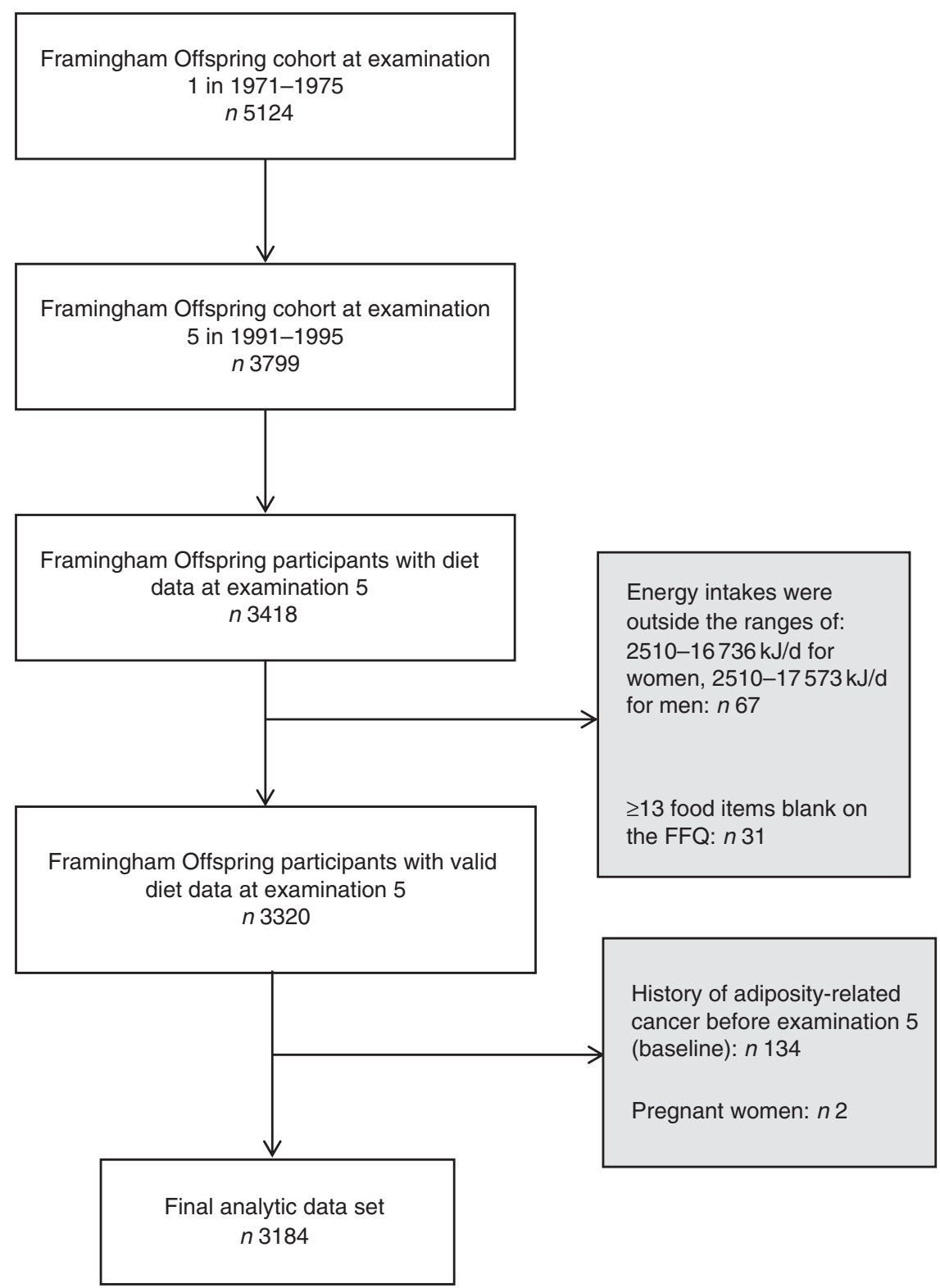

Fig. 1. Creation of the final analytical data set from the Framingham Offspring cohort. There were 5124 participants at examination 5 of whom 3418 had diet data collected. After excluding participants with invalid energy intakes $(n 67)^{(24)}$ and those who left $\geq 13$ food items blank on the FFQ $(n 31)^{(24)}$, there were 3320 participants with valid dietary data. Participants with a history of adiposity-related cancer at or before examination 5 ( $n$ 134) and pregnant women at examination 5 ( $n 2$ ) were also excluded resulting in the final analytic data set of 3184 participants.

FFQ (19\%) contain little or no carbohydrate and were thus excluded from the analyses. As previously published ${ }^{(28)}$, the average dietary GI was pre-calculated in the FHS as follows: $\{\Sigma[$ (Frequency of food per day) $\times$ (carbohydrate content of the food $) \times(G I)]\} /$ total carbohydrate in the diet.

Glycaemic load. The GL, a related concept, is a measure of both carbohydrate quantity and quality ${ }^{(13)}$. GL was computed for each food item on the FFQ by FHS investigators by multiplying the amount of available carbohydrate in the food by its GI and then dividing by $100^{(28)}$. The average dietary GL for each participant was then obtained by multiplying the carbohydrate content of each food by its GI and then multiplying this value by the frequency of consumption and summing up for all food items ${ }^{(28)}$. Potatoes, cold cereal, white bread, pizza, pasta, dark bread, orange juice, bananas, English muffins/bagels and white rice were the major foods contributing to the overall dietary GL within the FOS cohort, as previously published ${ }^{(28)}$.

Assessment of low-, medium- and high-glycaemic index foods. We classified carbohydrate-containing foods as high-, moderate- and low-GI foods if their GI was $\geq 70$, 56-69 and $\leq 55$, respectively ${ }^{(29)}$. Foods that are considered to be the main contributors of dietary carbohydrate intake in the US based on the National Health and Nutrition Examination Survey ${ }^{(30)}$ were selected a priori for the analyses. 


\section{Cancer case ascertainment}

The primary outcome for this study is adiposity-related cancers. These include cancers of the gastrointestinal tract, reticuloendothelial system (blood, bone and spleen), female reproductive tracts, genitourinary organs and the thyroid gland ${ }^{(2,3)}$. Cancers were considered adiposity-related if identified by the American Cancer Society or the National Cancer Institute as clearly or possibly linked to overweight and obesity ${ }^{(2,3)}$.

The FHS cancer cases include confirmed primary cancers from pathology reports with information on cancer type and date of diagnosis obtained from the patient's medical record. Cancer cases were ascertained using pathology reports with some diagnoses $(<5 \%)$ based solely on death certificates or clinical reports without pathology reports. Self-reported or suspected diagnoses not confirmed by pathology reports were excluded. A total of 699 adiposity-related cancers occurred in the FOS. After deleting participants with a history of adiposityrelated cancer at or before examination 5 ( $n$ 134), a total of 565 primary adiposity-related cancer cases (255 among women and 310 among men) including 124 breast, 157 prostate and sixty-eight colorectal cancers were identified.

\section{Measurement of other variables}

Demographic and lifestyle information. During in-person interviewing at clinical examination 5, age was self-reported; years of education were reported at examination 2. Smoking status, physical activity levels and alcohol use were also selfreported during in-person interviewing at the clinical examinations. Lifestyle information from examination 5 was used for these analyses. Participants were categorised as: current, former and non-smokers based on their smoking history. To assess their habitual physical activity levels, participants were asked to report the number of hours per week they spent engaging in sleep, sedentary, light, moderate or heavy physical activity on an average day ${ }^{(31)}$. The time spent engaging in these activities was then multiplied by their metabolic cost and summed to compute a physical activity index $(\mathrm{PAI})^{(31)}$. Alcohol intake (ounces/week) was computed from self-reported frequency of consumption of a standard serving of beer, wine and cocktails.

Anthropometric measures. Anthropometric measures including height, weight and waist circumference (WC) were measured with the subject standing by trained personnel at examination 5. BMI in $\mathrm{kg} / \mathrm{m}^{2}$ was calculated as follows: $\left(\mathrm{BMI}=\right.$ weight $(\mathrm{kg}) /$ height $\left.\left(\mathrm{m}^{2}\right)\right)$. Participants were considered 'normal', 'overweight' and 'obese' if their BMI was <25, 25-29.9 and $\geq 30 \mathrm{~kg} / \mathrm{m}^{2}$, respectively ${ }^{(32)}$. For WC measurements, men and women with $\mathrm{WC}>40$ and $>35$ inches, respectively, were considered 'at risk' (33).

Medical history. Participants were considered to have a history of chronic disease based on the presence or absence of diabetes and CVD at or before examination 5. Participants were considered to have diabetes if their fasting blood glucose was $\geq 7 \mathrm{mmol} / \mathrm{l}$ or if they were receiving diabetes treatment. Participants were considered to have CVD, as previously defined by $\mathrm{FHS}^{(34)}$. Among women, menopausal status, age at menopause and number of live births were determined using a standardised medical history questionnaire. Hormone therapy (HT) use was ascertained by the examining physician at this clinical examination.

\section{Statistical analysis}

Descriptive statistics were generated to examine clinical, demographic and lifestyle characteristics at examination 5 for the sample as a whole. These characteristics were also examined across the quintiles of total carbohydrate intake, expressed as percentage of energy intake, using general linear models procedure (PROC GLM). Cox proportional hazards models were used to calculate the hazard ratios (HR) and 95\% CI for total carbohydrate intake (\% energy), GI and GL $(\mathrm{g} / \mathrm{d})$ in relation to adiposity-related cancers with individuals in the lowest quintile category of the various dietary carbohydrate exposures as the referent category. Similarly, for low-, medium- and highGI foods, quintile categories were compared. The test for linear contrast was used to compute $P_{\text {trend }}$ for the detection of a linear trend across the quintiles of the carbohydrate exposure variables.

We also evaluated associations in relation to site-specific cancers (breast, prostate and colorectal cancers). Tertiles were created for the carbohydrate exposure variables in the sitespecific analyses because of the limited number of site-specific cancers in this cohort. We report herein the results of these exploratory analyses with the caveat of limited power. In all analyses, participants were considered censored if they died, were lost to follow-up or at the last examination in which they participated if the event had not yet occurred.

HR were adjusted for clinically important variables including age, sex, alcohol, smoking and energy intake ${ }^{(14)}$, which were selected a priori. We further adjusted for menopausal status, age at menopause, HT use and number of live births for breast cancer and for red and processed meat and fibre intake for colorectal cancer. For all analyses, we then tested other potential confounders including history of CVD or diabetes, physical activity, education, height and nutritional covariates such as fruit and vegetable and fat intake and use of antioxidant supplements ${ }^{(14)}$. These covariates were added singly to the model and were retained in the final models if they had an impact of $>10 \%$ on HR.

To determine whether BMI and WC are confounders or modify the impact of carbohydrate nutrition on risk of adiposity-related cancers, models were fitted with and without BMI and WC and analyses were also re-ran by BMI ('normal' $v$. 'overweight and obese') and WC strata ('normal' $v$. 'at risk'). We also tested for interactions with sex, physical activity and smoking because of their potential influence on the cancer process through various biological mechanisms, which may cause the risk estimates to vary ${ }^{(14)}$. A multiplicative term was introduced for these potential interactions in each model. $P_{\text {interaction }}<0 \cdot 1$ were considered significant, and if present, results were reported separately in subgroups. Statistical analyses were conducted using SAS statistical software (version 9.3; SAS Institute). 


\section{Results}

\section{Characteristics of the study population}

Table 1 represents the population characteristics evaluated at examination 5, which corresponds to the first period of dietary data collection. The FHS population is predominantly Caucasian $(99 \%)$, and $53.1 \%$ are females. On average, participants reported 14 years of education; mean age was 54.4 years and the mean BMI was $27.4 \mathrm{~kg} / \mathrm{m}^{2}$, indicating that the study sample, was middle aged to older and overweight. WC was within the normal range in both men and women ${ }^{(33)}$. The average PAI was $34 \cdot 8$, which represents a relatively high level of physical $\operatorname{activity}^{(31)}$. Approximately 43 and 19\% identified as former or current smokers, respectively. More than a third of the study population reported use of antioxidant supplements $(36 \cdot 2 \%)$.

Table 1. Baseline characteristics of Framingham Offspring cohort at examination 5 ( $n$ 3184)

(Percentages and numbers; mean values and standard deviations)

\begin{tabular}{|c|c|c|c|c|}
\hline Clinical characteristics & $\%$ & $n$ & Mean & SD \\
\hline Age (years) & & & 54.4 & $9 \cdot 7$ \\
\hline Education (years) (high school=12) & & & $14 \cdot 1$ & $2 \cdot 6$ \\
\hline Female (\%) & 53.1 & 1689 & & \\
\hline Physical activity index* & & & 34.8 & $6 \cdot 2$ \\
\hline $\mathrm{BMI}\left(\mathrm{kg} / \mathrm{m}^{2}\right)$ & & & $27 \cdot 4$ & 4.9 \\
\hline \multicolumn{5}{|l|}{ Waist circumference (inches) } \\
\hline Men & & & $39 \cdot 0$ & $4 \cdot 2$ \\
\hline Women & & & 34.3 & $5 \cdot 7$ \\
\hline \multicolumn{5}{|l|}{ Smoking status (\%)† } \\
\hline Never smoker & 37.3 & 1184 & & \\
\hline Former smoker & 43.3 & 1372 & & \\
\hline Current smoker & $19 \cdot 4$ & 615 & & \\
\hline \multicolumn{5}{|l|}{ Menopausal status (\%) } \\
\hline Postmenopausal & 63.9 & 1071 & & \\
\hline HT use (\%) & 19.5 & 330 & & \\
\hline Antioxidant use (\%)‡ & $36 \cdot 2$ & 1153 & & \\
\hline \multicolumn{5}{|l|}{ Dietary characteristics } \\
\hline Total energy content (kJ) & & & $7817 \cdot 0$ & $2612 \cdot 9$ \\
\hline Total energy content (kcal) & & & $1868 \cdot 3$ & $624 \cdot 5$ \\
\hline Total carbohydrates (\% energy) & & & $50 \cdot 9$ & 8.4 \\
\hline Total protein (\% energy) & & & $16 \cdot 8$ & $3 \cdot 3$ \\
\hline Total fat (\% energy) & & & $27 \cdot 4$ & 6.0 \\
\hline Glycaemic index & & & 54.7 & 3.5 \\
\hline Glycaemic load & & & $128 \cdot 8$ & 51.4 \\
\hline Fruits and vegetables (servings/d) & & & 3.7 & $2 \cdot 3$ \\
\hline Legumes (servings/week) & & & $2 \cdot 4$ & $2 \cdot 0$ \\
\hline Whole grains (oz eq/d) & & & 1.2 & 1.2 \\
\hline Refined grains (oz eq/d) & & & $4 \cdot 1$ & 1.7 \\
\hline $\begin{array}{l}\text { Red and processed meat } \\
\text { (servings/week)§ }\end{array}$ & & & $5 \cdot 3$ & 4.4 \\
\hline Alcohol (oz/week)\| & & & 2.5 & 3.8 \\
\hline
\end{tabular}

HT, hormone therapy.

* Physical activity index was computed as follows: $(1.0 \times$ hours of sleep $)+(1.1 \times$ hours of sedentary time $)+(1.5 \times$ hours of light physical activity $)+(2.4 \times$ hours of moderate activity $)+(5 \times$ hours of heavy physical activity).

† Current smokers: reported smoking at least one or more cigarettes per day regularly during the year preceding examination 5 ; former smokers: denied having smoked regularly for the year preceding the examination, but reported regular smoking more than 1 year before the examination; never smokers: did not report smoking at this or any previous clinical examination.

‡ Use of antioxidant supplements including vitamin A, vitamin C, vitamin E, Se, $\beta$-carotene was reported on the FFQ.

$\S$ Red and processed meat intake includes intake of bacon, hotdogs, processed meat, hamburger, meat sandwich and meat casserole.

II Alcohol intake in ounces per week was subsequently computed using the following equation: $(0.57 \times$ number of cocktails $)+(0.444 \times$ number of beers $)+(0.4 \times$ number of glasses of wine).
Among women, approximately 64\% were postmenopausal and $19.5 \%$ reported use of HT. The mean energy intake was $7816 \mathrm{~kJ}$, with carbohydrates accounting for approximately half of total energy intake ( $51 \%$ ). The average GI was $54 \cdot 7$, representing a low dietary GI, and the average GL was $128.8 \mathrm{~g} / \mathrm{d}$. On average, participants consumed 3.7 servings of fruits and vegetables/d, 2.4 servings/week of legumes and 5.3 servings/week of red and processed meat. The average intake of refined grains ( $4.1 \mathrm{oz} \mathrm{eq} / \mathrm{d})$ was more than double that of whole grains $(1.2 \mathrm{oz} \mathrm{eq} / \mathrm{d})$. On average, participants consumed 2.5 ounces of alcohol per week.

Associations of total carbohydrate intake, glycaemic index and glycaemic load and overall adiposity-related cancer risk

After a median follow-up of $13 \cdot 1$ years, there was no significant association between GI and GL in relation to overall adiposityrelated cancer risk (non-significant HR ranging from 0.93 to 0.95 ) in models adjusted for age, sex, smoking, alcohol and energy intake (Table 2). Additional adjustment for BMI, WC, history of CVD and diabetes, height, education, use of antioxidant supplements, physical activity, fruit and vegetable intake and fat intake did not significantly change these results. Total carbohydrate intake, expressed as percentage of energy intake, was associated with $30 \%$ lower risk of adiposity-related cancers in age-adjusted models (HR 0.70; 95\% CI 0.53, 0.92) $\left(P_{\text {trend }}=0.04\right)$, but this association was not statistically significant in multivariable-adjusted models (HR 0.77; 95\% CI $0.58,1.04)\left(P_{\text {trend }}=0.24\right)$.

Next, we tested for interactions for the relationships between dietary carbohydrates, GI and GL in relation to adiposity-related cancer risk, a priori considered significant at $P<0 \cdot 1$. There were no statistically significant interactions by BMI status $(P>0 \cdot 36)$, smoking $(P>0 \cdot 12)$ and by physical activity levels $(P>0.70)$ for any of the dietary variables. A significant multiplicative interaction was observed for sex and total carbohydrate intake (\% energy) $(P=0.08)$ and WC for all dietary variables $(P \leq 0.02)$. Stratified analyses by sex ('male' and 'female') and by WC ('normal' and 'at risk') did not reveal any significant associations (data not shown).

Associations of total carbohydrate intake, glycaemic index and glycaemic load with site-specific cancer risk

Breast cancer. After adjustment for age, smoking, alcohol, energy intake, menopausal status, HT use, age at menopause and number of live births, carbohydrate intake, as percentage of total energy intake, was associated with $41 \%$ lower risk of breast cancer (HR 0.59; 95\% CI 0.36, 0.97) (Table 3). Additional adjustment for height, pre-existing conditions (diabetes and CVD), antioxidant supplement use, education and physical activity did not change these findings. However, these associations were no longer significant in models additionally adjusted for BMI (HR 0.64; $95 \%$ CI $0.39,1.05)$ and WC (HR 0.63; $95 \%$ CI 0.38, 1.04). A null association was observed for GI and GL in relation to breast cancer risk in age- and multivariable-adjusted models (non-significant HR ranging from 0.54 to $0 \cdot 91$ ). 
Table 2. Association between quintiles of dietary carbohydrates, glycaemic index and glycaemic load in relation to adiposity-related cancer risk ( $n$ 656)

(Hazard ratios (HR) and $95 \%$ confidence intervals)

\begin{tabular}{|c|c|c|c|c|c|c|}
\hline & \multirow[b]{2}{*}{ No. at risk } & \multirow[b]{2}{*}{ No. with outcome } & \multicolumn{2}{|c|}{ Age adjusted } & \multicolumn{2}{|c|}{ Multivariable adjusted } \\
\hline & & & $\mathrm{HR}$ & $95 \% \mathrm{Cl}$ & $\mathrm{HR}^{*}$ & $95 \% \mathrm{Cl}$ \\
\hline \multicolumn{7}{|c|}{ Total carbohydrate intake (\% energy) } \\
\hline$<43.7$ & 644 & 128 & \multicolumn{2}{|c|}{1.00} & \multicolumn{2}{|c|}{1.00} \\
\hline $43 \cdot 7-48 \cdot 8$ & 630 & 110 & 0.87 & $0.68,1 \cdot 11$ & 0.92 & $0.70,1 \cdot 19$ \\
\hline $48 \cdot 8-52 \cdot 9$ & 637 & 118 & 0.87 & $0.67,1.11$ & 0.97 & $0.74,1.26$ \\
\hline $52 \cdot 9-57 \cdot 8$ & 639 & 116 & 0.94 & $0.73,1.21$ & 1.03 & $0.79,1.36$ \\
\hline$>57 \cdot 8$ & 634 & 93 & 0.70 & $0.53,0.92$ & 0.77 & $0.58,1.04$ \\
\hline$P_{\text {trend }}$ & & & \multicolumn{2}{|c|}{0.04} & \multicolumn{2}{|c|}{0.24} \\
\hline \multicolumn{7}{|l|}{ Glycaemic index } \\
\hline$<51.9$ & 626 & 114 & \multicolumn{2}{|c|}{1.00} & \multicolumn{2}{|c|}{1.00} \\
\hline $51 \cdot 9-54 \cdot 1$ & 660 & 115 & 0.85 & $0.66,1.11$ & 0.88 & $0.67,1.15$ \\
\hline $54 \cdot 1-55 \cdot 8$ & 638 & 107 & 0.88 & $0.68,1.15$ & 0.95 & $0.72,1.25$ \\
\hline $55 \cdot 8-57.5$ & 623 & 106 & 0.95 & $0.73,1 \cdot 24$ & 1.01 & $0.77,1.32$ \\
\hline$>57.5$ & 635 & 122 & 0.89 & $0.69,1 \cdot 16$ & 0.95 & $0.73,1.24$ \\
\hline$P_{\text {trend }}$ & & & \multicolumn{2}{|c|}{0.68} & \multicolumn{2}{|c|}{0.92} \\
\hline \multicolumn{7}{|c|}{ Glycaemic load (g/d) } \\
\hline$<85.6$ & 633 & 109 & \multicolumn{2}{|c|}{1.00} & \multicolumn{2}{|c|}{1.00} \\
\hline $85 \cdot 6-109 \cdot 5$ & 640 & 117 & 1.02 & $0.78,1.32$ & 1.03 & $0.78,1.36$ \\
\hline $109.5-133.3$ & 636 & 95 & 0.78 & $0.59,1.03$ & 0.78 & $0.57,1.07$ \\
\hline $133 \cdot 3-169 \cdot 9$ & 635 & 126 & 0.94 & $0.72,1.21$ & 0.94 & $0.70,1.33$ \\
\hline$>169.9$ & 638 & 117 & 0.97 & $0.75,1.26$ & 0.93 & $0.58,1.49$ \\
\hline$P_{\text {trend }}$ & & & \multicolumn{2}{|c|}{0.64} & \multicolumn{2}{|c|}{0.68} \\
\hline
\end{tabular}

* Models were adjusted for age, sex, smoking, alcohol, and energy (multivariable method for glycaemic index and glycaemic load).

Prostate cancer. There was no significant association between carbohydrate intake, dietary GI and GL in relation to prostate cancer risk (Table 3) (non-significant $\mathrm{HR}$ ranging from 0.74 to 0.99 ) in models adjusted for age and for smoking, alcohol and energy intake. Additional adjustment for BMI, WC, height, pre-existing conditions (CVD and diabetes), antioxidant use, education and physical activity did not alter any of these findings.

Colorectal cancer. Similarly, to the findings for prostate cancer, a null association was observed for total carbohydrate intake (\% energy), GI and GL in relation to colorectal cancer risk (Table 3) (non-significant HR ranging from 0.63 to 1.51) in models adjusted for age and for sex, smoking, alcohol, energy, red and processed meat and fibre intake. Additional adjustment for BMI, WC, height, pre-existing conditions (CVD and diabetes), antioxidant use, education and physical activity did not alter any of these findings.

\section{Associations of low-, medium- and high-glycaemic index carbohydrate food sources with adiposity-related and site-specific cancer risk}

In analyses evaluating low-, medium- and high-GI foods in relation to overall adiposity-related cancers and the examined site-specific cancers, there were no significant associations between high- and medium-GI foods and the risk of any cancer (Table 4). However, consumption of low-GI foods (including most fruits and non-starchy vegetables, legumes, milk and dairy products and whole grain products), collectively, in the highest $v$. lowest tertile of intake was associated with $49 \%$ lower breast cancer risk (HR 0.51; 95\% CI 0.32, 0.83) in multivariableadjusted models.

Although low-GI foods, collectively, were not significantly associated with adiposity-related cancer risk, among individual low-GI foods, consumption of legumes was also associated with $36 \%$ lower risk of adiposity-related cancers combined (HR 0.64; $95 \%$ CI $0.47,0.88) \quad(P=0.009)$. A statistically significant multiplicative interaction was observed for legumes with sex $(P=0 \cdot 005)$. In sex-stratified analyses, associations were only significant among women, as consumption of legumes in the highest $v$. lowest quintile was associated with $43 \%$ lower risk of adiposity-related cancers (HR 0.57; $95 \% \mathrm{CI}$ $0.35,0.91)\left(P_{\text {trend }}=0.02\right)$.

\section{Discussion}

In this cohort of aging Caucasian adults, GI and GL were not associated with risk of adiposity-related cancers combined or any of the site-specific cancers. Higher carbohydrate consumption was associated with $41 \%$ lower risk of breast cancer, but this association disappeared after adjustment for measures of body adiposity. Among low-, medium- and high-GI foods, low-GI foods were collectively associated with $49 \%$ lower primary incidence of breast cancer. Although no significant associations were observed between individual low-GI foods and breast cancer risk, higher consumption of legumes was associated with $36 \%$ lower risk of adiposity-related cancers combined. The protective impact of legumes was more pronounced among women, for whom legume consumption was associated with $43 \%$ lower risk of adiposity-related cancers. There was no association between any of the carbohydrate food sources with prostate and colorectal cancer. 
Table 3. Association between tertiles (T) of dietary carbohydrates, glycaemic index (Gl) and glycaemic load (GL) in relation to breast, prostate and colorectal cancers (Hazard ratios (HR) and $95 \%$ confidence intervals)

\begin{tabular}{|c|c|c|c|c|c|c|c|c|c|c|c|c|c|c|c|c|c|c|}
\hline & \multicolumn{6}{|c|}{ Breast cancer $(n 124)^{\star} \dagger \ddagger$} & \multicolumn{6}{|c|}{ Prostate cancer $(n$ 157)§†॥ } & \multicolumn{6}{|c|}{ Colorectal cancer $\left(n\right.$ 68) $\boldsymbol{T}^{\star \star *}$} \\
\hline & \multirow[b]{2}{*}{ At risk } & \multirow[b]{2}{*}{ Cancer cases } & \multicolumn{2}{|c|}{ Age adjusted } & \multicolumn{2}{|c|}{$\begin{array}{l}\text { Multivariable- } \\
\text { adjusted }\end{array}$} & \multirow[b]{2}{*}{ At risk } & \multirow[b]{2}{*}{ Cancer cases } & \multicolumn{2}{|c|}{ Age adjusted } & \multicolumn{2}{|c|}{$\begin{array}{l}\text { Multivariable } \\
\text { adjusted }\end{array}$} & \multirow[b]{2}{*}{ At risk } & \multirow[b]{2}{*}{ Cancer cases } & \multicolumn{2}{|c|}{ Age adjusted } & \multicolumn{2}{|c|}{$\begin{array}{l}\text { Multivariable } \\
\text { adjusted }\end{array}$} \\
\hline & & & $\mathrm{HR}$ & $95 \% \mathrm{Cl}$ & $\mathrm{HR}^{\star}$ & $95 \% \mathrm{Cl}$ & & & $\mathrm{HR}$ & $95 \% \mathrm{Cl}$ & HR§ & $95 \% \mathrm{Cl}$ & & & $\mathrm{HR}$ & $95 \% \mathrm{Cl}$ & $\mathrm{HR}$ & $95 \% \mathrm{Cl}$ \\
\hline \multicolumn{19}{|c|}{ Carbohydrate intake (\% energy) } \\
\hline $\mathrm{T} 1$ & 558 & 48 & & 1.00 & & 1.00 & 492 & 55 & & 1.00 & & 1.00 & 1049 & 27 & & 1.00 & & 1.00 \\
\hline T2 & 573 & 45 & 0.80 & $0.53,1.20$ & 0.84 & $0.55,1.30$ & 506 & 50 & 0.83 & $0.56,1.22$ & 0.85 & $0.57,1.27$ & 1079 & 23 & 0.75 & $0.43,1.31$ & 1.26 & $0.68,2.34$ \\
\hline T3 & 558 & 31 & 0.62 & $0.39,0.97$ & 0.59 & $0.36,0.97$ & 497 & 52 & 0.92 & $0.62,1.35$ & 0.95 & $0.62,1.45$ & 1056 & 18 & 0.63 & $0.35,1.16$ & 1.45 & $0.70,3.04$ \\
\hline \multicolumn{19}{|c|}{ 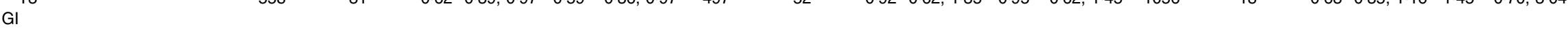 } \\
\hline $\mathrm{T} 1$ & 551 & 48 & & 1.00 & & 1.00 & 486 & 53 & & 1.00 & & 1.00 & 1059 & 18 & & 1.00 & & 1.00 \\
\hline T2 & 572 & 31 & 0.65 & $0.42,1.03$ & 0.67 & $0.42,1.06$ & 522 & 62 & 1.09 & $0.75,1.57$ & 1.06 & $0.73,1.55$ & 1071 & 26 & 1.41 & $0.78,2.58$ & 1.61 & $0.87,2.98$ \\
\hline T3 & 566 & 45 & 0.91 & $0.61,1.37$ & 0.90 & $0.59,1.37$ & 485 & 41 & 0.74 & $0.49,1 \cdot 12$ & 0.74 & $0.48,1 \cdot 12$ & 1052 & 24 & 1.28 & $0.69,2.35$ & 1.51 & $0.81,2.84$ \\
\hline \multicolumn{19}{|c|}{$0.14 \quad 0.49,1.12 \quad 0.14 \quad 0.40,1.12$} \\
\hline $\mathrm{T} 1$ & 557 & 46 & & 1.00 & & 1.00 & 494 & 48 & & 1.00 & & 1.00 & 1051 & 25 & & 1.00 & & 1.00 \\
\hline T2 & 575 & 44 & 0.87 & $0.58,1.32$ & 0.75 & $0.47,1.22$ & 506 & 58 & 1.24 & $0.84,1.82$ & 1.08 & $0.70,1.68$ & 1079 & 25 & 0.91 & $0.52,1.58$ & 1.19 & $0.61,2 \cdot 30$ \\
\hline T3 & 557 & 34 & 0.69 & $0.44,1.07$ & 0.54 & $0.26,1.09$ & 493 & 50 & 0.99 & $0.67,1.48$ & 0.76 & $0.40,1.43$ & 1052 & 18 & 0.66 & $0.36,1.21$ & 1.21 & $0.43,3.40$ \\
\hline
\end{tabular}

* For breast cancer, models were adjusted for age, smoking, alcohol, energy (multivariable method for Gl and GL), menopausal status, hormone therapy use, age at menopause and number of live births.

† Additional adjustment for BMI, waist circumference, height, pre-existing conditions (diabetes and CVD), antioxidant supplement use, education, and physical activity did not change these findings. An exception was the model on carbohydrate inake (\% energy) in relation to beast cancer

\%: for Gl: T1: <53.3, T2: 53.3-56.2, T3: >56.2; for GL (g/d): T1: <96.7 g/d, T2: 96.7-136.0 g/d, T3: >136.0 g/d.

I The tertile cut-offs for prostate cancer were: carbohydrate intake (\% energy): T1: <46.2\%, T2: 46.2-53.7\%, T3: >53.7\%; for Gl: T1: $<53 \cdot 6$, T2: 53.6-56.4, T3: > 56.4; for GL (g/d): T1: <106.3 g/d, T2: 106.3-154.4 g/d, T3: >154.4 g/d.

For colorectal cancer, models were adjusted for age, sex, smoking, alcohol, energy (multivariable method for Gl and GL), red and processed meat intake and fibre intake.
The tertile cut-offs for colorectal cancer were: carbohydrate intake (\% energy): T1: $<47 \cdot 3 \%$, T2: 47.3-54.4\%, T3: >54.4\%; for Gl: T1: $<53 \cdot 5$, T2: 53.5-56.3, T3: $>56 \cdot 3$; for GL: T1: < 100.7 g/d, T2:100.7-143.7 g/d, T3: > 143.7 g/d. 
Table 4. Association between quintiles $(\mathrm{Q})$ /tertiles $(\mathrm{T})$ of low-, medium-, and high-glycaemic index (Gl) foods (servings/week) in relation to overall adiposity-related cancers and site-specific cancers (Hazard ratios (HR) and $95 \%$ confidence intervals)

\begin{tabular}{|c|c|c|c|c|c|c|c|c|c|c|c|c|c|c|c|c|}
\hline \multirow[b]{3}{*}{ Carbohydrate-containing foods } & \multicolumn{4}{|c|}{ Adiposity-related cancers ${ }^{\star} † \ddagger$} & \multicolumn{4}{|c|}{ Breast cancers§†॥ } & \multicolumn{4}{|c|}{ Prostate cancers $\boldsymbol{\Psi} \boldsymbol{\dagger}^{\star *}$} & \multicolumn{4}{|c|}{ 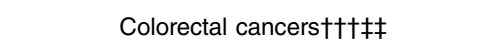 } \\
\hline & \multicolumn{2}{|c|}{ Age adjusted } & \multicolumn{2}{|c|}{ Multivariable adjusted $^{*} \dagger$} & \multicolumn{2}{|c|}{ Age adjusted } & \multicolumn{2}{|c|}{ Multivariable adjusted§† } & \multicolumn{2}{|c|}{ Age adjusted } & \multicolumn{2}{|c|}{ Multivariable adjusted $\uparrow \dagger$} & \multicolumn{2}{|c|}{ Age adjusted } & \multicolumn{2}{|c|}{ Multivariable adjusted $\dagger \dagger$} \\
\hline & $\mathrm{HR}$ & $95 \% \mathrm{Cl}$ & $\mathrm{HR}$ & $95 \% \mathrm{Cl}$ & $\mathrm{HR}$ & $95 \% \mathrm{Cl}$ & $\mathrm{HR}$ & $95 \% \mathrm{Cl}$ & $\mathrm{HR}$ & $95 \% \mathrm{Cl}$ & $\mathrm{HR}$ & $95 \% \mathrm{Cl}$ & $\mathrm{HR}$ & $95 \% \mathrm{Cl}$ & $\mathrm{HR}$ & $95 \% \mathrm{Cl}$ \\
\hline \multicolumn{17}{|l|}{ Low-Gl foods $\S$} \\
\hline Category 1 & \multicolumn{2}{|r|}{1.00} & \multicolumn{2}{|r|}{1.00} & \multicolumn{2}{|r|}{1.00} & \multicolumn{2}{|c|}{1.00} & \multicolumn{2}{|r|}{1.00} & \multicolumn{2}{|c|}{1.00} & \multicolumn{2}{|r|}{1.00} & \multicolumn{2}{|c|}{1.00} \\
\hline Category 2 & 0.91 & $0.71,1.18$ & 0.95 & $0.73,1.23$ & 0.61 & $0.40,0.93$ & 0.59 & $0.38,0.92$ & $1 \cdot 15$ & $0.78,1.71$ & 1.14 & $0.75,1.71$ & 0.44 & $0.24,0.81$ & 0.52 & $0.27,0.99$ \\
\hline Category 3 & 0.79 & $0.61,1.03$ & 0.80 & $0.61,1.06$ & 0.56 & $0.37,0.87$ & 0.51 & $0.32,0.83$ & $1 \cdot 16$ & $0.78,1.73$ & 1.16 & $0.75,1.81$ & 0.57 & $0.32,1.00$ & 0.76 & $0.38,1.51$ \\
\hline Category 4 & 0.85 & $0.65,1 \cdot 10$ & 0.85 & $0.65,1.12$ & & - & & - & & - & & - & & - & & - \\
\hline Category 5 & 0.80 & $0.61,1.04$ & 0.80 & $0.59,1.08$ & & - & & - & & - & & - & & - & & - \\
\hline \multicolumn{17}{|l|}{ Medium-GI foodsIIII } \\
\hline Category 1 & \multicolumn{2}{|r|}{1.00} & \multicolumn{2}{|r|}{1.00} & \multicolumn{2}{|r|}{1.00} & \multicolumn{2}{|c|}{1.00} & \multicolumn{2}{|r|}{1.00} & \multicolumn{2}{|c|}{1.00} & \multicolumn{2}{|r|}{1.00} & & 1.00 \\
\hline Category 2 & 0.89 & $0.68,1 \cdot 15$ & 0.94 & $0.72,1.22$ & 0.99 & $0.64,1.53$ & 1.04 & $0.66,1.63$ & 1.20 & $0.81,1.79$ & 1.17 & $0.77,1.76$ & 0.95 & $0.54,1.65$ & 1.19 & $0.66,2 \cdot 15$ \\
\hline Category 3 & 0.82 & $0.63,1.08$ & 0.85 & $0.64,1.12$ & 1.08 & $0.70,1.67$ & 1.26 & $0.77,2.04$ & $1 \cdot 15$ & $0.78,1.69$ & 1.13 & $0.73,1.75$ & 0.65 & $0.36,1 \cdot 19$ & 1.03 & $0.48,2 \cdot 24$ \\
\hline Category 4 & 0.89 & $0.69,1 \cdot 16$ & 0.89 & $0.67,1.18$ & & - & & - & & - & & - & & - & & - \\
\hline Category 5 & 0.86 & $0.67,1.12$ & 0.87 & $0.64,1 \cdot 17$ & & - & & - & & - & & - & & - & & - \\
\hline $\begin{array}{l}P_{\text {trend }} \\
\text { Hiah-Gl foods II }\end{array}$ & & 0.32 & & 0.33 & & - & & - & & - & & - & & - & & - \\
\hline Category 1 & & 1.00 & & 1.00 & & 1.00 & & 1.00 & & 1.00 & & 1.00 & & 1.00 & & 1.00 \\
\hline Category 2 & 0.85 & $0.65,1 \cdot 10$ & 0.93 & $0.70,1.23$ & 0.75 & $0.49,1 \cdot 14$ & 0.72 & $0.45,1.16$ & 1.24 & $0.84,1.85$ & 1.28 & $0.83,1.99$ & 0.89 & $0.51,1.55$ & 1.14 & $0 \cdot 61,2 \cdot 13$ \\
\hline Category 3 & 0.83 & $0.64,1.09$ & 0.90 & $0.67,1.22$ & 0.70 & $0.45,1.08$ & 0.64 & $0.34,1.24$ & 1.26 & $0.84,1.88$ & 1.40 & $0.78,2.53$ & 0.65 & $0.35,1.21$ & 0.95 & $0.39,2 \cdot 31$ \\
\hline Category 4 & 0.97 & $0.75,1.26$ & 1.04 & $0.75,1.43$ & & - & & - & & - & & - & & - & & - \\
\hline Category 5 & 0.91 & $0.70,1.18$ & 0.99 & $0.66,1.49$ & & - & & - & & - & & - & & - & & - \\
\hline$P_{\text {trend }}$ & & 0.88 & & 0.85 & & - & & - & & - & & - & & - & & - \\
\hline
\end{tabular}

* Models were adjusted for age, sex, smoking, alcohol and energy.

† Additional adjustment for BMI, waist circumference, height, pre-existing conditions (diabetes and CVD), antioxidant supplement use, education and physical activity did not change these findings and mutual adjustment of low-, medium, and high-Gl for each other did not change these findings.

: Wh-GI foods (servings/week): Q1: <15.7, Q2: 15.7-22.8, Q3: 22.8-29.4, Q4: 29.4-39.5, Q5: >39.5; for medium-GI foods: Q1: <5.5, Q2: 5.5-8.7, Q3: 8.7-11.9, Q4: 11.9-16.8, , for high-Gl foods: Q1: <33.7, Q2: 33.7-45.2, Q3: 45.2-57.6, Q4: 57.6-75.0, Q5: > 75.0.

§ For breast cancer, models were adjusted for age, smoking, alcohol, energy (multivariable method), menopausal status, hormone therapy use, age at menopause and number of live births.
I The tertile cut-offs for breast cancer were: low-Gl foods (servings/week): T1: $<21.9$, T2: 21.9-33.8, T3: $>33 \cdot 8$; for medium-Gl foods (servings/week): T1: $<7 \cdot 8$, T2: 7.8-13.4, T3: $>13 \cdot 4$; high-Gl foods: T1: $<40 \cdot 6$, T2: 40.6-59.8, T3: $>59 \cdot 8$. I For prostate cancer, models were adjusted for age, smoking, alohol and energy (mutivarible method).

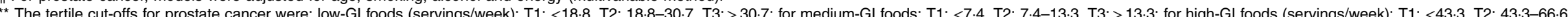
T3: > 66.6.

t† For colorectal cancer, models were adjusted for age, sex, smoking, alcohol, energy (multivariable method), red and processed meat intake and fibre intake.

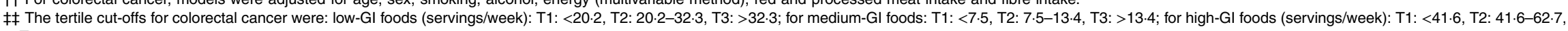
T3: >62.7.

$\S \S$ Low-Gl foods included most fruits, non-starchy vegetables and carrots, legumes and certain whole grain and dairy products.

IIII Medium-GI foods included certain fruits (e.g. bananas and blueberries), whole grain products (e.g. popcorn and cereal), desserts and baked goods and sugary drinks (e.g. juice and soft drinks).

IT High-Gl foods included certain fruits (e.g. cantaloupe and watermelons), fast foods (French fries and doughnuts), potatoes, white bread, cold cereal and candy. 
Null findings for dietary carbohydrates, GI and GL in relation to adiposity-related cancers are consistent with the literature, as most studies on this topic report null or weak associations $^{(17-20,35,36)}$. Evidence on carbohydrate food sources in relation to cancer risk is limited. Our finding that legumes significantly reduce cancer risk is biologically plausible by virtue of their fibre, folate and phytochemical content, which possess anti-carcinogenic properties ${ }^{(14,37)}$. This result is also consistent with previous literature showing a more pronounced benefit among women ${ }^{(37,38)}$, as legumes are a concentrated source of phytoestrogens, namely isoflavones, which have weak oestrogenic properties and possess putative antioestrogenic effects that may reduce the risk of female adiposity-related cancers. However, this finding of a protective effect of low-GI foods, particularly legumes, on cancer risk may be because of the chance given the multiple comparisons conducted in these analyses.

For gastrointestinal cancers, a review of case-control evidence was suggestive of a detrimental impact of GI and GL intake in some studies ${ }^{(17)}$. However, pooled cohort study results showed no associations between total carbohydrate intake, GI and GL and the risk of these cancers ${ }^{(17,19,20)}$, particularly colorectal cancer ${ }^{(17,19)}$. Although diets high in foods containing dietary fibre such as fruits, vegetables, legumes and whole grains have been convincingly linked to colorectal cancer ${ }^{(14)}$, we did not find any significant associations between carbohydrate-containing foods and colorectal cancer risk. However, this may be because of the limited number of colorectal cancer cases in this cohort, as the strength of associations between nutritional factors and cancer is moderate making it difficult to detect associations in studies with limited power.

For prostate cancer, our null findings for total carbohydrate intake, GI and GL are consistent with the limited epidemiologic evidence summarised in recent meta-analysis of case-control and prospective cohort studies, which was also indicative of a null association ${ }^{(36)}$. Our finding of a null association between high-GI foods such as fast foods, and soft drinks and prostate cancer risk is inconsistent with case-control evidence suggestive of up to $64 \%$ higher aggressive prostate cancer risk with higher consumption of high-GI foods ${ }^{(39)}$, but is consistent with results from a recent Swedish study, which reported a null association between soft drinks and prostate cancer ${ }^{(40)}$.

For breast cancer, a recent systematic review and metaanalysis reported an overall null association between GI and GL and the risk of breast cancer ${ }^{(41)}$, which is consistent with the findings in these analyses. In contrast, for total carbohydrate intake and breast cancer, our results from models unadjusted for measures of body adiposity are inconsistent with most cohort studies, which report a null association ${ }^{(42)}$. Some studies have even documented a detrimental impact of $>2$-fold higher breast cancer risk with higher dietary carbohydrate intake ${ }^{(43,44)}$. Nevertheless, an analysis within the Nurses Health Study ${ }^{(45)}$ showed that dietary carbohydrates were associated $38 \%$ lower breast cancer risk among women with BMI $<25 \mathrm{~kg} / \mathrm{m}^{2}$, but we were unable to investigate the impact of dietary carbohydrates on breast cancer risk by BMI status in this study because of the limited number of breast cancer cases. Lastly, we also documented that low-GI foods are associated with decreased breast cancer risk. Studies on carbohydrate-containing foods and breast cancer are limited, primarily to evidence of a moderate but significant protective impact of fruits and vegetables ${ }^{(14,46)}$. However, this finding of a protective effect of low-GI foods on breast cancer risk may be because of the chance given the multiple comparisons conducted in these analyses and the limited number of breast cancer cases.

It is notable that the inverse association between dietary carbohydrate intake and breast cancer risk in this cohort was attenuated and was no longer statistically significant upon adjustment for BMI and WC, suggesting that these measures of body adiposity may explain the observed association. In fact, in this cohort, participants with higher carbohydrate intake had significantly lower BMI $(P<0.0001)$ and WC $(P \leq 0.0238)$ (online Supplementary Table S1) potentially resulting in a lower risk of cancer. These findings suggest that the influence of overweight and obesity on breast cancer risk is likely more pronounced than that of dietary carbohydrates and are comparable with previous evidence showing strong associations between body adiposity and cancers compared with the other examined site-specific cancers.

Beyond BMI and WC, the discrepancy between the inverse associations in our study and the null results in most existing studies on total carbohydrate intake and breast cancer may be ascribed to the lifestyle differences among participants in the highest $v$. lowest category of carbohydrate intake in this cohort (online Supplementary Table S1). We found that participants in the highest quintile of carbohydrate intake reported higher intakes of fruits and vegetables, legumes and whole grains, and higher antioxidant supplement use, which may collectively reduce the risk of excess adiposity and protect against cancer $^{(14)}$. They also had a lower percentage of smokers and reported lower intakes of red and processed meat and alcohol, all of which are potential cancer risk factors ${ }^{(14)}$. In particular, up to $10 \%$ higher breast cancer risk has been reported when comparing women in the highest $v$. the lowest category of red and processed meat intake ${ }^{(47)}$. Similarly, women who are moderate and heavy drinkers exhibit $4 \%$ and $40-50 \%$ higher risk of developing breast cancer compared with non-drinkers ${ }^{(48)}$. Therefore, the observed inverse associations may be a marker of healthier dietary and lifestyle habits among participants with higher carbohydrate intakes in this cohort. Finally, it is possible that the observed association between dietary carbohydrates and breast cancer was because of the chance, particularly given the limited number of breast cancer cases in this cohort.

Some study limitations deserve mention. The lack of an association between GI and GL in relation to cancer may be attributed, in part, to the FFQ used to assess dietary intakes. This FFQ was not specifically developed to measure GI and GL, and the food items listed in the FFQ may not capture the full detectable range of GI and GL. This may have resulted in random misclassification of the exposures and underestimated the associations with cancer risk. In addition, self-reported intakes measured by FFQ in observational studies are generally prone to underreporting, which may have biased associations towards the null ${ }^{(49)}$. Methodological limitations in the measurement of dietary GI and GL for individual foods may have 
also influenced the observed associations. Reference GI values from both Australian and American foods were used to estimate dietary GI and GL, but differences in processing, cooking methods and dietary intakes can have a significant impact on the GI of these foods ${ }^{(13)}$. There is also suggestion that GI and GL may not accurately capture the glycaemic or insulinaemic response to food when used in the context of a usual diet ${ }^{(50,51)}$, which may account, at least in part, for the observed null associations. Power was limited to evaluate associations for individual cancer sites, and particularly for subtypes within these cancers (e.g. hormone receptor status for breast cancer). Furthermore, the FHS cancer file did not include information on men with a history of prostatectomy for exclusion from the analytical data set.

Another limitation of this study is that the diet and lifestyle variables assessed at examination 5 were considered for these analyses, which may not capture changes over time or perhaps during the relevant critical window of exposure that affects cancer risk. Our previous report on trends in dietary carbohydrate consumption in this cohort indicated that there were no clinically significant changes intakes of carbohydrates and their food sources over the study time frame (1991-2008) ${ }^{(23)}$. Therefore, for dietary variables, it is unlikely that use of information from one time point significantly altered the results. Moreover, although we adjusted for many relevant confounders, it is possible that residual confounding occurred because of unrecognised factors or errors in assessing these covariates.

This study has a number of notable strengths. The prospective study design allows avoidance of the typical limitations inherent to case-control studies including recall and selection bias, inability to establish temporality and to estimate disease incidence. Another unique strength is the long duration of follow-up of more than two decades (approximately 22 years). Furthermore, the study used a validated tool for dietary assessment, reliable anthropometric measures obtained by trained personnel, and confirmation of cancer cases using medical records and pathology reports. In addition, a wide range of information on potential confounders and effectmodifiers was available and accounted for in these analyses.

In conclusion, carbohydrate quantity and quality were not associated with combined incidence of adiposity-related cancers, though carbohydrate quantity was associated with reduced breast cancer risk. An investigation of the role of carbohydrate foods sources suggested that women in particular might benefit from a dietary pattern that emphasises healthier, low-GI carbohydrate-containing foods, particularly legumes. Given the existing methodological issues in measuring GI and the inconsistent evidence from epidemiological studies, there is a lack of consensus on the role of GI and GL in chronic disease prevention. However, the American Diabetes Association recommends choosing low-GI foods as means to refine diabetes management that is complimentary to carbohydrate counting $^{(29)}$. For adiposity-related cancers in particular, the WCRF/AICR deemed evidence on GI and cancer insufficient for incorporation of this concept into cancer prevention guidelines ${ }^{(14,52)}$. Additional research is needed to clarify the role of a low-GI and GL diet in the risk of various site-specific cancers within diverse ethnic groups, as an aetiologic agent and not just a marker of a healthy diet.

\section{Acknowledgements}

The authors thank the FHS and dbGaP for providing access to the FHS data sets.

The FHS is conducted and supported by the National Heart, Lung, and Blood Institute (NHLBI) in collaboration with Boston University (contract no. N01-HC-25 195). Funding support for the Framingham FFQ data sets was provided by USDA Agricultural Research Service contract no. 53-3k06-5-10, USDA Agricultural Research Service agreement nos 58-1950-9-001, 58-1950-4-401 and 58-1950-7-707. This manuscript was not prepared in collaboration with the core team of FHS investigators and does not necessarily reflect the opinions or views of the FHS, Boston University or NHLBI. The present study was supported by the American Cancer Society Research Scholar Grant (no. RSG-12-005-01-CNE) awarded to Dr N. P. The American Cancer Society had no role in the design and analysis of the study or in the writing of this article.

The authors' contributions are as follows: N. M. and N. P. conceived this project and developed the overall research plan. N. M. took the lead in writing the manuscript and conducted the statistical analyses; Y. L. advised on the statistical analyses and reviewed the manuscript for the statistical accuracy and interpretation of results; E. V. B., P. F. J. and N. P. provided insights into the analytical plan and review and revision of the manuscript for important intellectual content; N. P. reviewed the manuscript for important intellectual content and had primary responsibility for the final content and for overseeing the entire study.

The authors declare that there are no conflicts of interest.

\section{Supplementary material}

For supplementary material/s referred to in this article, please visit https://doi.org/10.1017/S0007114517001489

\section{References}

1. Siegel RL, Miller KD \& Jemal A (2017) Cancer statistics, 2017. CA Cancer J Clin 67, 7-30.

2. National Cancer Institute (2014) Obesity and cancer risk. http://www.cancer.gov/cancertopics/factsheet/Risk/obesity (accessed April 2017).

3. American Cancer Society (2013) Body weight and cancer risk. http://www.cancer.org/cancer/cancercauses/dietandphysical activity/bodyweightandcancerrisk/body-weight-and-cancerrisk-effects (accessed April 2017).

4. Yabroff KR, Lund J, Kepka D, et al. (2011) Economic burden of cancer in the United States: estimates, projections, and future research. Cancer Epidemiol Biomarkers Prev 20, 2006-2014.

5. Parekh N, Lin Y, Vadiveloo M, et al. (2013) Metabolic dysregulation of the insulin-glucose axis and risk of obesityrelated cancers in the Framingham heart study-offspring cohort (1971-2008). Cancer Epidemiol Biomarkers Prev 22, $1825-1836$. 
6. Roberts DL, Dive C \& Renehan AG (2010) Biological mechanisms linking obesity and cancer risk: new perspectives. Annu Rev Med 61, 301-316.

7. Doyle SL, Donohoe CL, Lysaght J, et al. (2012) Visceral obesity, metabolic syndrome, insulin resistance and cancer. Proc Nutr Soc 7, 181-189.

8. Shikata K, Ninomiya T \& Kiyohara Y (2013) Diabetes mellitus and cancer risk: review of the epidemiological evidence. Cancer Sci 104, 9-14.

9. Te Morenga L, Mallard S \& Mann J (2013) Dietary sugars and body weight: systematic review and meta-analyses of randomized controlled trials and cohort studies. BMJ 346, e 7492.

10. Santos F, Esteves S, da Costa Pereira A, et al. (2012) Systematic review and meta-analysis of clinical trials of the effects of low carbohydrate diets on cardiovascular risk factors. Obes Rev $\mathbf{1 3}$ 1048-1066.

11. Jenkins DJ, Wolever TM, Taylor RH, et al. (1981) Glycemic index of foods: a physiological basis for carbohydrate exchange. Am J Clin Nutr 34, 362-366.

12. Jenkins DA, Jenkins A, Wolever TS, et al. (1984) The glycaemic response to carbohydrate foods. Lancet 324, 388-391.

13. Atkinson FS, Foster-Powell K \& Brand-Miller JC (2008) International tables of glycemic index and glycemic load values: 2008. Diabetes Care 31, 2281-2283.

14. American Institute for Cancer Research \& World Cancer Research Fund (2007) Food, Nutrition, and Physical Activity and the Prevention of Cancer: A Global Perspective. Washington, DC: American Institute for Cancer Research.

15. Dong J \& Qin L (2011) Dietary glycemic index, glycemic load, and risk of breast cancer: meta-analysis of prospective cohort studies. Breast Cancer Res Treat 126, 287-294

16. Gnagnarella P, Gandini S, La Vecchia C, et al. (2008) Glycemic index, glycemic load, and cancer risk: a meta-analysis. $\mathrm{Am}$ J Clin Nutr 87, 1793-1801.

17. Mulholland HG, Murray LJ, Cardwell CR, et al. (2009) Glycemic index, glycemic load, and risk of digestive tract neoplasms: a systematic review and meta-analysis. Am J Clin Nutr 89, 568-576.

18. Nagle CM, Olsen CM, Ibiebele TI, et al. (2012) Glycemic index, glycemic load and endometrial cancer risk: results from the Australian National Endometrial Cancer study and an updated systematic review and meta-analysis. Eur J Nutr $\mathbf{5 2}$, $1-11$.

19. Aune D, Chan D, Lau R, et al. (2012) Carbohydrates, glycemic index, glycemic load, and colorectal cancer risk: a systematic review and meta-analysis of cohort studies. Cancer Cause Control 23, 521-535.

20. Aune D, Chan D, Vieira A, et al. (2012) Dietary fructose, carbohydrates, glycemic indices and pancreatic cancer risk: a systematic review and meta-analysis of cohort studies. Ann Oncol 23, 2536-2546.

21. Dawber TR, Meadors GF \& Moore FE Jr (1951) Epidemiological approaches to heart disease: the Framingham Study. Am J Public Health 41, 279-281.

22. Feinleib M, Kannel WB, Garrison RJ, et al. (1975) The Framingham offspring study. Design and preliminary data. Prev Med 4, 518-525.

23. Makarem N, Scott M, Quatromoni P, et al. (2014) Trends in dietary carbohydrate consumption from 1991 to 2008 in the Framingham Heart Study Offspring Cohort. BrJ Nutr 27, 1-14.

24. McKeown NM, Meigs JB, Liu S, et al. (2004) Carbohydrate nutrition, insulin resistance, and the prevalence of the metabolic syndrome in the Framingham Offspring Cohort. Diabetes Care 27, 538-546.

25. Rimm EB, Giovannucci EL, Stampfer MJ, et al. (1992) Reproducibility and validity of an expanded self-administered semiquantitative food frequency questionnaire among male health professionals. Am J Epidemiol 135, 1114-1126.

26. Willett WC, Sampson L, Stampfer MJ, et al. (1985) Reproducibility and validity of a semiquantitative food frequency questionnaire. Am J Epidemiol 122, 51-65.

27. Liu S, Manson JE, Stampfer MJ, et al. (2001) Dietary glycemic load assessed by food-frequency questionnaire in relation to plasma high-density-lipoprotein cholesterol and fasting plasma triacylglycerols in postmenopausal women. Am J Clin Nutr 73, 560-566.

28. McKeown NM, Meigs JB, Liu S, et al. (2009) Dietary carbohydrates and cardiovascular disease risk factors in the Framingham offspring cohort. J Am Coll Nutr 28, 150-158.

29. American Diabetes Association (2014) Glycemic index and diabetes. http://www.diabetes.org/food-and-fitness/food/whatcan-i-eat/understanding-carbohydrates/glycemic-index-anddiabetes.html (accessed April 2017).

30. Block G (2004) Foods contributing to energy intake in the US: data from NHANES III and NHANES 1999-2000. J Food Comp Anal 17, 439-447.

31. Kannel WB \& Sorlie P (1979) Some health benefits of physical activity: the Framingham Study. Arch Intern Med 139, 857.

32. World Health Organization (2016) BMI classification. http:// apps.who.int/bmi/index.jsp?introPage=intro_3.html (accessed April 2017).

33. National Heart, Lung, and Blood Institute (2016) Guidelines on overweight and obesity: according to waist circumference. http://www.nhlbi.nih.gov/health-pro/guidelines/current/ obesity-guidelines/e_textbook/txgd/4142.htm (accessed April 2017).

34. Hubert HB, Feinleib M, McNamara PM, et al. (1983) Obesity as an independent risk factor for cardiovascular disease: a 26-year follow-up of participants in the Framingham Heart Study. Circulation 67, 968-977.

35. Nagle CM, Kolahdooz F, Ibiebele TI, et al. (2011) Carbohydrate intake, glycemic load, glycemic index, and risk of ovarian cancer. Ann Oncol 22, 1332-1338.

36. Zhai L, Cheng S \& Zhang D (2015) Dietary carbohydrate and prostate cancer risk: a meta-analysis. Nutr Cancer $\mathbf{6 7}$, 594-602.

37. Messina MJ (1999) Legumes and soybeans: overview of their nutritional profiles and health effects. Am J Clin Nutr 70, 439S-450SS

38. Farvid MS, Cho E, Chen WY, et al. (2014) Dietary protein sources in early adulthood and breast cancer incidence: prospective cohort study. BMJ 348, g3437.

39. Hardin J, Cheng I \& Witte JS (2011) Impact of consumption of vegetable, fruit, grain, and high glycemic index foods on aggressive prostate cancer risk. Nutr Cancer 63, 860-872.

40. Drake I, Sonestedt E, Gullberg B, et al. (2012) Dietary intakes of carbohydrates in relation to prostate cancer risk: a prospective study in the Malmö Diet and Cancer cohort. Am J Clin Nutr 96, 1409-1418.

41. Mullie P, Koechlin A, Boniol M, et al. (2016) Relation between Breast cancer and high glycemic index or glycemic load: a meta-analysis of prospective cohort studies. Crit Rev Food Sci Nutr 56, 152-159

42. Michels KB, Mohllajee AP, Roset-Bahmanyar E, et al. (2007) Diet and breast cancer. Cancer 109, 2712-2749.

43. Wen W, Shu XO, Li H, et al. (2009) Dietary carbohydrates, fiber, and breast cancer risk in Chinese women. Am J Clin Nutr 89, 283-289.

44. Romieu I, Lazcano-Ponce E, Sanchez-Zamorano LM, et al. (2004) Carbohydrates and the risk of breast cancer among Mexican women. Cancer Epidemiol Biomarkers Prev 13, $1283-1289$. 
45. Cho E, Spiegelman D, Hunter DJ, et al. (2003) Premenopausal dietary carbohydrate, glycemic index, glycemic load, and fiber in relation to risk of breast cancer. Cancer Epidemiol Biomarkers Prev 12, 1153-1158.

46. Albuquerque RC, Baltar VT \& Marchioni DM (2014) Breast cancer and dietary patterns: a systematic review. Nutr Rev $\mathbf{7 2}$, $1-17$.

47. Guo J, Wei W \& Zhan L (2015) Red and processed meat intake and risk of breast cancer: a meta-analysis of prospective studies. Breast Cancer Res Treat 151, 191-198.

48. Seitz HK, Pelucchi C, Bagnardi V, et al. (2012) Epidemiology and pathophysiology of alcohol and breast cancer: update 2012. Alcohol Alcohol 47, 204-212.

49. Schatzkin A, Kipnis V, Carroll RJ, et al. (2003) A comparison of a food frequency questionnaire with a 24-hour recall for use in an epidemiological cohort study: results from the biomarkerbased Observing Protein and Energy Nutrition (OPEN) study. Int J Epidemiol 32, 1054-1062.

50. Holt SH, Miller JC \& Petocz P (1997) An insulin index of foods: the insulin demand generated by $1000-\mathrm{kJ}$ portions of common foods. Am J Clin Nutr 66, 1264-1276.

51. Mayer-Davis EJ, Dhawan A, Liese AD, et al. (2006) Towards understanding of glycemic index and glycemic load in habitual diet: associations with measures of glycaemia in the Insulin Resistance Atherosclerosis Study. Br J Nutr 95 , 397-405.

52. American Institute for Cancer Research (2016) The glycemic index: what it is and what it is not. http://preventcancer.aicr. org/site/DocServer/Glycemic_Index.pdf?docID=341 (accessed April 2017). 